\title{
Comparação de dois tratamentos fisioterapêuticos na redução da dor e aumento da autonomia funcional de idosos com gonartrose
}

\author{
Comparison of two physiotherapy treatments in reducing pain and increasing functional autonomy \\ of elderly patients with gonarthrosis
}

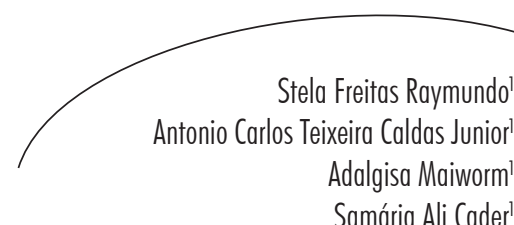

Resumo

Objetivo: Comparar o tratamento da terapia manual, associada ao ultrassom, com o tratamento da cinesioterapia convencional, associada ao laser de arseneto de galium (AsGa), na redução da dor e no aumento da autonomia funcional em idosos com gonartrose. Métodos: Estudo clínico, de delineamento quase-experimental. A amostra foi dividida aleatoriamente em dois grupos: grupo experimental 1 (GE1; $n=44$; idade $=68 \pm 7,64$ anos; índice de massa corporal: $26,77 \pm 4,74 \mathrm{Kg} / \mathrm{m}^{2}$ ) - idosos com gonartrose submetidos ao tratamento fisioterapêutico com terapia manual e ultrassom; e grupo experimental 2 (GE2; $n=20$; idade $=70 \pm 5,49$ anos; índice de massa corporal: $\left.27,11 \pm 4,63 \mathrm{Kg} / \mathrm{m}^{2}\right)$ - idosos com gonartrose submetidos ao tratamento fisioterápico com cinesioterapia convencional e laserterapia. Foram analisados a autonomia funcional (protocolo do Grupo de Desenvolvimento Latino-Americano para a Maturidade GDLAM) e o quadro álgico (escala CR10 de Borg). Ambos os GEs participaram de sessões de treinamento com duração de 45 minutos, duas vezes semanais, durante oito semanas consecutivas. O nível de significância foi de $p<0,05$. Resultados: Observouse melhora da autonomia funcional pela redução significativa do índice de GDLAM $(\mathrm{p}<0,001)$ tanto no GE1 $(\Delta=16,14)$ quanto no GE2 $(\Delta=9,32)$. Semelhantemente, houve diminuição significativa $(\mathrm{p}<0,001)$ do nível de dor tanto no GE1 $(\Delta=5,09)$ quanto no GE2 $(\Delta=7,1)$. O poder do experimento foi de $98 \%$. Conclusão: Infere-se, assim, que ambos os tratamentos sugeridos neste estudo são eficazes no aumento da autonomia funcional e na redução do quadro álgico de idosos com gonartrose.

Abstract

Objective: To compare the treatment of manual therapy associated with ultrasound, and conventional kinesiotherapy associated with gallium arsenide laser (GaAs) in reducing pain and increasing functional autonomy in elderly patients with gonarthrosis. Methods: Clinical trial, with quasi-experimental design. The sample was randomly divided into two groups: experimental group 1 (EG1, $n=44$, age $=68 \pm 7.64$ years, body mass index:

Divisão de Fisioterapia, Policlínica Piquet Carneiro. Universidade do Estado do Rio de Janeiro. Rio de Janeiro, RJ, Brasil.

Correspondência / Correspondence

Samária Ali Cader

E-mail: samariacader@gmail.com

Palavras-chave:

Manipulações

Musculoesqueléticas. Terapia por Ultrassom. Atividades Cotidianas. Osteoartrite do Joelho. Envelhecimento. 
$\left.26.77 \pm 4.74 \mathrm{~kg} / \mathrm{m}^{2}\right)$ - patients with gonarthrosis undergoing physical therapy with manual therapy and ultrasound; and experimental group 2 (EG2, $n=20$, age $70 \pm 5.49$ years, body mass index: $27.11 \pm 4.63 \mathrm{~kg} / \mathrm{m}^{2}$ ) - patients with gonarthrosis undergoing physical therapy and laser therapy with conventional kinesiotherapy. We analyzed functional autonomy (Protocol Development Group for Latin American Maturity - GDLAM) and pain symptoms (Borg CR10 scale). Both EGs participated in 45-minute training sessions, twice a week for eight consecutive weeks. The significance level was $\mathrm{p}<0.05$. Results: It was observed significant improvement in the reduction of the functional autonomy index GDLAM $(\mathrm{p}<0.001)$ in both EG1 $(\Delta=16.14)$ and in EG2 $(\Delta=9.32)$. Similarly, there was significant decrease $(\mathrm{p}<0.001)$ in the level of pain in both EG1 $(\Delta=5.09)$ and EG2 ( $\Delta=7.1)$. The power of the experiment was $98 \%$. Conclusions: Both treatments were effective in increasing the functional autonomy and reduction of pain in elderly patients with gonarthrosis.

\section{INTRODUÇÃO}

A osteoartrose $(\mathrm{OA})$, também chamada de doença articular degenerativa, é uma condição músculo-esquelética importante caracterizada pela perda da cartilagem articular que leva à dor e à perda de função. A articulação mais comumente afetada é o joelho, e a OA do joellho (gonartrose) pode resultar em mudanças que afetam não só tecidos intracapsulares, mas também periarticulares, como ligamentos, cápsulas, tendões e músculos. ${ }^{1}$ Em um esforço mais recente para definir OA, a European League Against Rheumatism (EULAR) sugeriu que um diagnóstico clínico confiável de OA do joelho pode ser feito de acordo com três sintomas (dor no joelho persistente, rigidez matinal e função reduzida) e três sinais (crepitação, de circulação restrita e alargamento ósseo). ${ }^{2}$

O tratamento da gonartrose é dirigido à redução da dor e rigidez nas articulações; manutenção e melhora da mobilidade articular; redução da incapacidade física, a qual limita as atividades da vida diária; melhora da qualidade de vida; limitação da progressão das lesões articulares; educação dos pacientes sobre a natureza da doença e seu tratamento. ${ }^{3}$ Society International (OARSI), diversas terapias não farmacológicas foram incluídas em suas diretrizes para o tratamento de OA de quadril e
No encontro de 2009 da Osteoarthritis Research
Key words: Musculoskeletal Manipulations. Ultrasonic Therapy. Activities of Daily Living. Osteoarthritis Knne. Aging. joelho: acupuntura; terapia manual; fisioterapia; dispositivos (incluindo órteses, calçados, andadores); educação e autocuidado; perda de peso; eletroterapia (TENS, ultrassom e laser); modalidades térmica; cirurgia e reabilitação. ${ }^{4}$

Em 1992 a Agência para a Política de Saúde e Pesquisa Clínica no Tratamento da Dor recomendou ambas abordagens cognitivocomportamentais (educação do paciente, simples relaxamento, biofeedback) e agentes terapêuticos e modalidades (calor superficial, frio, massagem, exercícios de imobilidade, eletro-analgesia) como essencial no tratamento da dor aguda. ${ }^{5}$ Além disso, várias diretrizes para o cuidado da $\mathrm{OA}$, incluindo as do American College of Rheumatology, ${ }^{6}$ a European League Against Rheumatism, ${ }^{7}$ Algorithms for the Diagnosis and Management of Musculoskeletal Complaints $^{8}$ e Institute for Clinical Systems Improvement ${ }^{9}$ recomendam recursos como o uso de gelo, calor e estimulação elétrica. ${ }^{10}$

A crioterapia envolve a aplicação de frio, utilizando sacos de gelo ou água gelada. O frio penetra nos tecidos moles e, quando aplicado sobre um conjunto, reduz a temperatura intraarticular, atrasando assim a realização de sinais nervosos e reduzindo o fluxo sanguíneo local. Essas mudanças, por sua vez, reduzem o sinal de transmissão de substâncias nocivas de resposta inflamatória e, posteriormente, afetam o nível de perda de sangue, inchaço local e dor percebida. ${ }^{11}$ 
A terapia manual, uma das recomendações descritas nas diretrizes da OARSI, é um tratamento físico utilizado pelos fisioterapeutas, quiropráticos, osteopatas e outros profissionais no tratamento da dor músculo-esquelética e deficiência, e inclui massagem terapêutica, mobilização articular e manipulação. 12,13 Outro tratamento físico é a cinesioterapia, dita convencional, um programa de treinamento cujo foco é melhorar a aptidão global geral, o equilíbrio corporal, a coordenação motora, a flexibilidade, a resistência e a força muscular dos membros inferiores, melhorando a função global e os sintomas de pacientes com OA. ${ }^{14}$

Neste sentido, esta investigação teve por objetivo comparar o tratamento da terapia manual, associada à utilização do aparelho de ultrassom com o tratamento da cinesioterapia convencional, associada à utilização do aparelho laser de arseneto de galium (AsGa) na redução da dor e aumento da autonomia funcional em idosos com gonartrose.

\section{MATERIAIS E MÉTODOS}

Amostra

O presente estudo clínico, de delineamento quase-experimental, teve o universo inicialmente constituído de 73 idosos com gonartrose, de ambos os sexos, encaminhados para a Divisão de Fisioterapia da Policlínica Piquet Carneiro no período de outubro de 2011 a setembro de 2012. A seleção da amostra foi realizada de forma randômica, por sorteio, sendo depurada, obedecendo aos critérios de inclusão e exclusão. Quando o paciente era convocado a iniciar o tratamento, realizava-se o sorteio por meio da retirada de um cartão, podendo este pertencer ao grupo experimental 1 ou 2.

Os voluntários deveriam atender aos critérios de inclusão: capacidade de realizar atividades da vida diária sem auxílio e não praticar outra modalidade de atividade física durante o estudo, de forma regular com controle de carga e intensidade, a fim de não interferir nos resultados. Os critérios de exclusão foram: presença de patologias que pudessem causar limitações físicas, para a realização dos testes de autonomia funcional, ou mentais, para responderem à escala de dor.

Após a depuração dos critérios de inclusão e exclusão, foram excluídos nove sujeitos; assim a amostra final $(\mathrm{n}=64)$ foi dividida randomicamente por sorteio, descrito anteriormente, em: grupo experimental (GE) 1, com idosos com gonartrose submetidos ao tratamento fisioterapêutico com terapia manual e ultrassom (GE1; $n=44$; idade $=68 \pm 7,64$ anos; índice de massa corporal: $\left.26,77 \pm 4,74 \mathrm{Kg} / \mathrm{m}^{2}\right)$, e grupo experimental 2 , com idosos com gonartrose submetidos ao tratamento fisioterapêutico com cinesioterapia convencional e laserterapia (GE2; $\mathrm{n}=20$; idade $=70 \pm 5,49$ anos; índice de massa corporal: $\left.27,11 \pm 4,63 \mathrm{Kg} / \mathrm{m}^{2}\right)$. Ambos os GEs realizaram seus respectivos protocolos com a frequência de duas vezes por semana, com duração de 45 minutos cada sessão, durante oito semanas.

O presente estudo atendeu às normas para a realização de pesquisa em seres humanos, Resolução no 196/96, do Conselho Nacional de Saúde, e da Resolução de Helsinki de 2008. O estudo teve seu projeto de pesquisa submetido ao Comitê de Ética em Pesquisa do Hospital Universitário Pedro Ernesto, tendo sido aprovado sob $\mathrm{n}^{\circ}$ CAAE: 0175.0.228.000-11. Todos os participantes do estudo assinaram o Termo de Consentimento Livre e Esclarecido.

\section{Procedimentos}

Inicialmente, todos os participantes passaram por uma anamnese, constando de questões referentes aos aspectos sociodemográficos (idade; sexo; raça/cor, estado civil, escolaridade, atividade laboral) e aos aspectos de saúde (medicamentos utilizados, presença ou não de doenças pulmonares, auditivos, visuais, diabetes, hipertensão arterial, fraturas, entre outras). 


\section{Avaliação do Índice de Massa Corporal}

A fim de manter homogeneidade da amostra, foi realizada avaliação da massa corporal e da estatura, para o cálculo do índice de massa corporal ${ }^{15}$ (balança com estadiômetro Filizola, 2007).

\section{Avaliação da Autonomia Funcional}

Avaliada por meio do protocolo do Grupo de Desenvolvimento Latino-Americano para a Maturidade (GDLAM), composto por cinco testes: a) caminhar $10 \mathrm{~m}(\mathrm{C} 10 \mathrm{~m})$ - o participante percorre uma distância de $10 \mathrm{~m}$ em determinado tempo; ${ }^{16}$ b) levantar-se da posição sentada (LPS), com os braços cruzados em frente ao corpo - o participante senta-se e levanta-se da cadeira cinco vezes ao comando do avaliador; ${ }^{17}$ c) levantar-se da posição decúbito ventral (LPDV) - é dado um comando ao participante, que deve levantarse do chão no menor tempo possível, saindo da posição decúbito ventral para a ortostase ${ }^{18}$ d) vestir e tirar uma camiseta (VTC) - ao comando do avaliador, o participante deverá vestir e tirar uma camiseta no menor tempo possível; ${ }^{19}$ e) levantar-se da cadeira e locomover-se pela casa (LCLC) - sentado com os pés fora do chão, o participante deve realizar um circuito em formato de triângulo, onde o mesmo contorna os cones dispostos a três metros da cadeira na diagonal direita e esquerda, voltando a sentar-se na mesma cadeira onde iniciou o teste, realizando o circuito duas vezes, no menor tempo possível. ${ }^{20}$

Após a realização dos testes, os valores são calculados por meio de uma fórmula matemática, para o cálculo do índice geral de GDLAM (IG). ${ }^{21}$ Cada teste recebe uma classificação que varia de fraco, regular, bom e muito bom (tabela 1).

\section{Avaliação da dor}

O nível de dor foi avaliado pela escala CR10 de Borg. ${ }^{22}$

Tabela 1. Padrão de avaliação da autonomia funcional do protocolo GDLAM. Rio de Janeiro-RJ, 2011-2012.

\begin{tabular}{lcccccc}
\hline $\begin{array}{l}\text { Classificação } \\
\text { dos testes }\end{array}$ & $\begin{array}{c}\text { C 10M } \\
(\text { seg.) }\end{array}$ & $\begin{array}{c}\text { LPS } \\
(\text { seg.) }\end{array}$ & $\begin{array}{c}\text { LPDV } \\
\text { (seg.) }\end{array}$ & $\begin{array}{c}\text { VTC } \\
(\text { seg. })\end{array}$ & $\begin{array}{c}\text { LCLC } \\
(\text { seg.) }\end{array}$ & $\begin{array}{c}\text { IG } \\
\text { (escores) }\end{array}$ \\
\hline Fraco & $+7,09$ & $+11,19$ & $+4,40$ & $+13,14$ & $+43,00$ & $+27,42$ \\
Regular & $7,09-6,34$ & $11,19-9,55$ & $4,40-3,30$ & $13,14-11,62$ & $43,00-38,69$ & $27,42-24,98$ \\
Bom & $6,33-5,71$ & $9,54-7,89$ & $3,29-2,63$ & $11,61-10,14$ & $38,68-34,78$ & $24,97-22,66$ \\
Muito bom & $-5,71$ & $-7,89$ & $-2,63$ & $-10,14$ & $-34,78$ & $-22,66$ \\
\hline
\end{tabular}

C10m= caminhar 10m; LPS= levantar-se da posição sentada; LPDV= levantar-se da posição decúbito ventral; VTC= vestir e tirar uma camiseta; LCLC= levantar-se da cadeira e locomover-se pela casa; seg.= valores em segundos; IG= índice de GDLAM (valores em escores).

\section{Tratamento fisioterapêutico}

Ultrassom associado à terapia manual (Muligan) GE1: quando necessário, antes da utilização da eletroterapia (ultrassom), era aplicada a crioterapia em um período de 20 minutos ao redor da articulação do joelho acometido. A utilização da eletroterapia foi realizada pelo ultrassom, com as seguintes características: forma de onda contínua; feixe ultrassônico, sem ocorrer pausa entre os feixes; dosimetria: média de 1,0 w/ $\mathrm{cm}^{2}$ (efeito anti-inflamatório, analgésico, regenerador e restauração óssea), método de deslizamento: transdutor fica em contato direto com a pele, com leve pressão sobre ela; tempo de aplicação de cinco minutos; uma aplicação diária, duas vezes por semana.

O método de terapia manual utilizado, Mulligan, consistiu em restaurar o alinhamento normal ou o trilho do movimento da 
articulação, com disfunção e, assim, recuperar a artrocinemática normal, resultando no ganho de amplitude de movimento e diminuição da dor; técnica utilizada com carga, sendo feita mobilizações com movimentos ativos ou passivos, que foram sustentados, havendo uma pressão mais intensa no final do arco. Foram realizadas nesses pacientes três séries de dez repetições.

\section{Tratamento fisioterapêutico}

\section{Laser associado à cinesioterapia convencional}

No GE2, a utilização da eletroterapia foi realizada pelo laser de arseneto de gálium (AsGa), com as seguintes características: forma de onda pulsada; feixe de luz não visível; dosimetria: média de 03 joules $/ \mathrm{cm}^{2}$ (efeito anti-inflamatório, circulatório, analgésico e regenerador); forma de deposição de energia: pontual; uma aplicação diária, duas vezes por semana; posicionamento da caneta a $90^{\circ}$.

Importante ressaltar que o ambiente deve ser fechado, com pouca luminosidade, baixo índice de reflexão do feixe de luz; ambos (o fisioterapeuta e o paciente) devem estar utilizando óculos específicos de proteção e a caneta deve ser envolvida com a mão. Após a aplicação do laser, antes da cinesioterapia convencional, caso houvesse necessidade, também foi utilizada a aplicação de crioterapia por 20 minutos ao redor do joelho acometido.

A cinesioterapia convencional seguiu o seguinte protocolo: alongamento passivo de toda musculatura de membros inferiores (MmIi); fortalecimento de quadríceps: agachamento uma série de 12 vezes mantendo ângulo de $30^{\circ}$ do joelho, extensão de joelhos com caneleira de $1 / 2 \mathrm{~kg}$ no tornozelo (três séries de 12 vezes), em posição sentada na maca e pernas pendentes; fortalecimento de panturrilhas com flexão plantar partindo de ortostatismo (duas séries de 12 vezes); treino de descida e subida da escada de dois degraus (uma série de 15 vezes); treino de equilíbrio unipodal em MmIi no solo; treino de subida e descida na rampa por um minuto com e sem apoio de membros superiores; flexão de quadril em ortostatismo com caneleira de $1 / 2 \mathrm{~kg}$ (uma série de 12 vezes) bipodal.

Na progressão da cinesioterapia: alongamento ativo-passivo de toda musculatura de MmIi; fortalecimento de quadríceps: agachamento duas séries de 12 vezes mantendo ângulo de $30^{\circ}$ do joelho, extensão de joelhos com caneleira de $1 \mathrm{~kg}$ no tornozelo (três séries de 12 vezes), em posição sentada na maca e pernas pendentes; fortalecimento de panturrilhas com flexão plantar partindo de ortostatismo (três séries de 12 vezes); treino de descida e subida da escada de dois degraus (duas séries de 15 vezes); treino de equilíbrio unipodal em MmIi no solo; treino de subida e descida na rampa por um minuto com e sem apoio de membros superiores; flexão de quadril em ortostatismo com caneleira de $1 \mathrm{~kg}$ (duas séries de 12 vezes) bipodal.

\section{Tratamento estatístico}

Os dados do estudo foram tratados pelo programa SPSS 20.0 para Windows e Microsoft Office Excel, versão 2010. Foram empregadas técnicas de estatística descritiva para caracterizar o universo amostral pesquisado. Para a descrição dos dados coletados, foram utilizados a média como medida de localização, e o desvio-padrão como medida de dispersão. Também foi calculado delta absoluto $(\Delta)$, por meio da subtração dos resultados da fase pré-teste - pós-teste. Para avaliar a normalidade da amostra, utilizou-se o teste de Shapiro Wilk. Para as comparações intra e intergrupos, foi empregado o teste de ANOVA twoway, seguido do post hoc de Scheffé para identificar as possíveis diferenças. O estudo admitiu o nível de $\mathrm{p}<0,05$ para a significância estatística.

\section{RESULTADOS}

Os dados descritivos e a análise de distribuição dos dados do grupo experimental 1 estão expostos na tabela 2. Pode-se observar que não houve distribuição normal nas variáveis LPS, VTC e LCLC. 
Tabela 2. Análise descritiva e inferencial de Shapiro Wilk do grupo experimental 1. Rio de Janeiro-RJ, 2011-2012.

\begin{tabular}{ccccc}
\hline & & Média & Desvio-padrão & SW \\
\hline \multirow{2}{*}{ C10m } & pré-teste & 16,59 & 4,12 & 0,144 \\
& pós-teste & 10,12 & 2,67 & \\
\multirow{2}{*}{ LPS } & pré-teste & 24,43 & 7,10 & $\mathbf{0 , 0 1 5}$ \\
& pós-teste & 15,53 & 4,98 & \\
\multirow{2}{*}{ VTC } & pré-teste & 19,03 & 5,54 & 0,152 \\
& pós-teste & 11,01 & 4,37 & \\
\multirow{2}{*}{ LCLC } & pré-teste & 19,68 & 4,15 & $\mathbf{0 , 0 0 7}$ \\
& pós-teste & 13,12 & 3,19 & $\mathbf{0 , 0 0 2}$ \\
\multirow{2}{*}{ IG } & pré-teste & 51,74 & 12,08 & \\
& pós-teste & 47,06 & 11,51 & 0,196 \\
\multirow{2}{*}{ Borg } & pré-teste & 52,80 & 7,80 & 0,200 \\
& pós-teste & 36,66 & 6,35 & \\
\hline
\end{tabular}

C10m= caminhar 10 metros; LPS= levantar da posição sentada; LPDV= levantar da posição de decúbito ventral; VTC= vestir e tirar uma camiseta; LCLC= levantar da cadeira e locomover-se pela casa; IG = índice de GDLAM; SW= p-valor do Shapiro Wilk. A unidade de medida dos testes é segundos.

Estão apresentados, na tabela 3, os dados descritivos e a análise de distribuição dos dados do grupo experimental 2. Pode-se observar que, com exceção do LCLC, houve distribuição normal de todas as variáveis. 
Tabela 3. Análise descritiva e inferencial de Shapiro Wilk do grupo experimental 2. Rio de Janeiro-RJ, 2011-2012.

\begin{tabular}{ccccc}
\hline & & Média & Desvio-padrão & SW \\
\hline \multirow{2}{*}{ C10m } & pré-teste & 15,58 & 2,69 & 0,153 \\
& pós-teste & 10,93 & 1,28 & \\
\multirow{2}{*}{ LPS } & pré-teste & 22,83 & 1,42 & 0,106 \\
& pós-teste & 18,87 & 0,45 & \\
LPDV & pré-teste & 17,36 & 1,20 & 0,498 \\
& pós-teste & 15,38 & 0,77 & 0,410 \\
VTC & pré-teste & 18,55 & 1,53 & \\
& pós-teste & 14,77 & 0,62 & $\mathbf{0 , 0 3 1}$ \\
LCLC & pré-teste & 47,98 & 4,78 & 0,196 \\
& pós-teste & 39,42 & 3,23 & \\
\multirow{2}{*}{ IG } & pré-teste & 49,15 & 3,27 & 0,200 \\
& pós-teste & 39,83 & 2,39 & \\
\hline \multirow{2}{*}{ Borg } & pré-teste & 7,82 & 1,30 & 1,18 \\
\end{tabular}

C10m= caminhar 10 metros; LPS= levantar da posição sentada; LPDV= levantar da posição de decúbito ventral; VTC= vestir e tirar uma camiseta; LCLC= levantar da cadeira e locomover-se pela casa; IG = índice de GDLAM; SW= p-valor do Shapiro Wilk. A unidade de medida dos testes é segundos.

Na figura 1 está representada a comparação intra e intergrupos da autonomia funcional por meio do delta absoluto ( $\Delta=$ pré-teste - pós-teste). $\mathrm{Na}$ comparação intragrupos, pode-se verificar melhora significativa do teste C10m (GE1 e GE2: $p<0,001$ ), LPS (GE1: $\mathrm{p}<0,001)$, LPDV (GE1: $p<0,001)$, VTC (GE1: $p<0,001 ;$ GE2: $\mathrm{p}=0,004)$ e IG (GE1 e GE2: $\mathrm{p}<0,001)$. Na análise intergrupos, houve apenas diferença significativa na variável LPDV ( $p=0,003$ ), a favor do GE1, na fase pós-teste. $\mathrm{Na}$ comparação intergrupos na fase pré-teste, não houve diferença significativa entre os grupos. 


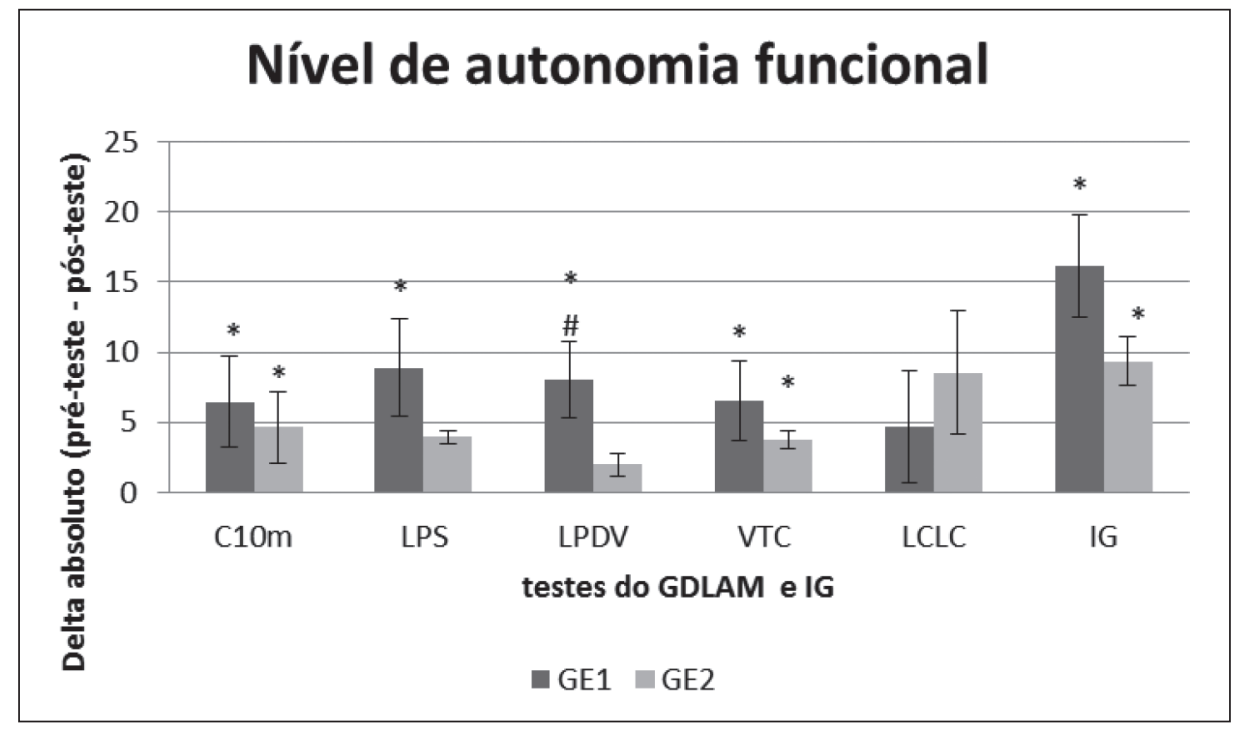

${ }^{*} \mathrm{p}<0,05$ (pré-teste x pós-teste); \#p<0,05 (pós-teste GE1 x pós-teste GE2).

GE1= grupo experimental 1; GE2 = grupo experimental 2; C10m= caminhar 10 metros; LPS= levantar da posição sentada; LPDV= levantar da posição de decúbito ventral; VTC= vestir e tirar uma camiseta; LCLC= levantar da cadeira e locomover-se pela casa; IG= índice de GDLAM. A unidade de medida dos testes é segundos.

Figura 1. Comparação intra e intergrupos da autonomia funcional. Rio de Janeiro-RJ, 2011-2012.

A comparação intra e intergrupos do nível de dor, por meio do delta absoluto, está exposta na figura 2. Na comparação intragrupos, observouse redução significativa na fase pós-teste
( $\mathrm{p}<0,001) . \mathrm{Na}$ análise intergrupos, não houve diferença significativa na fase pré-teste e nem na pós-teste $\mathrm{O}$ poder do experimento encontrado neste estudo foi de $98 \%$.

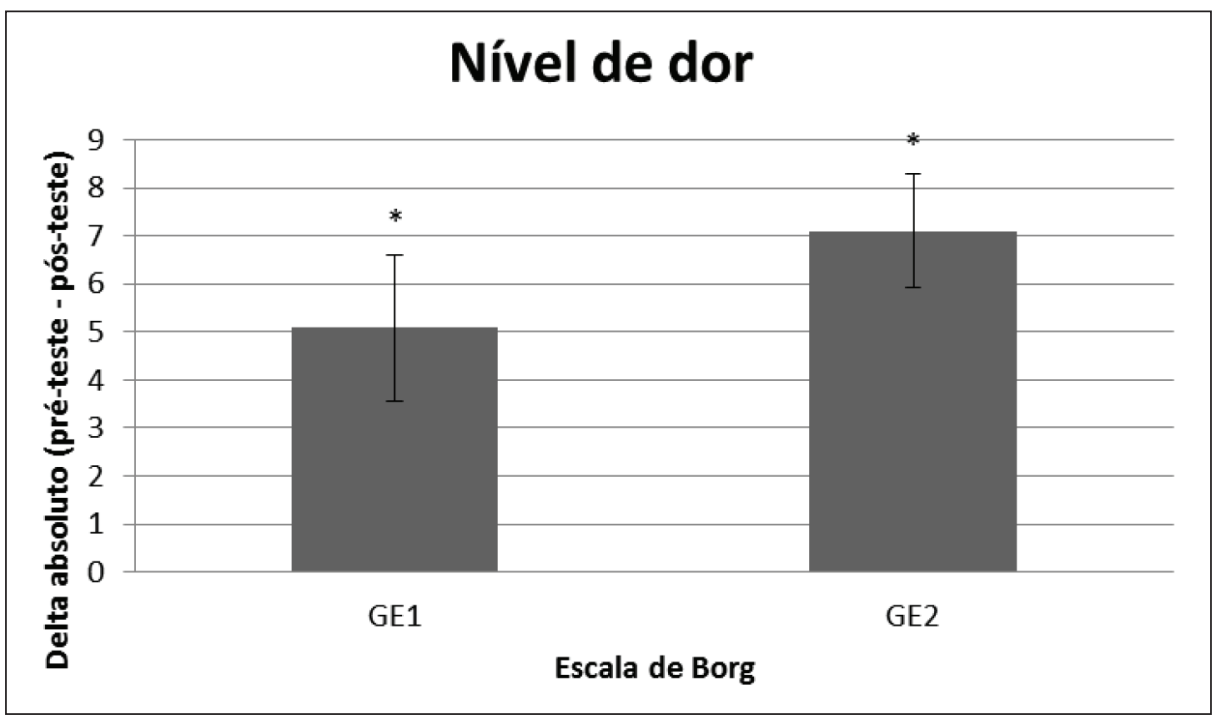

*p<0,05 (pré-teste x pós-teste); GE1= grupo experimental 1; GE2= grupo experimental 2.

Figura 2. Comparação intra e intergrupos do nível de dor. Rio de Janeiro-RJ, 2011-2012. 


\section{DISCUSSÃO}

As principais modalidades terapêuticas usadascomumente na prática da fisioterapia são calor, crioterapia, eletroterapia e cinesioterapia. ${ }^{23}$ $\mathrm{O}$ uso dessas técnicas para diminuir a dor e acelerar a cicatrização é uma prática secular que ainda é adequada hoje em dia. Particularmente no espectro da gestão da dor, multiformas de terapia, incluindo educação psicológica do paciente, exercício e atividade física, cinesioterapia, tratamento farmacológico e intervenções cirúrgicas, devem completar um ao outro para o máximo benefício ao paciente.

Na população geriátrica, modalidades físicas, quando prescritas em um programa de terapia, devem ser a base do tratamento da dor e da disfunção física, especialmente quando a causa é uma condição músculo-esquelética. Nos idosos, isto se torna mais relevante uma vez que a dependência exclusiva da farmacoterapia no tratamento da dor e disfunção tem muitas desvantagens. ${ }^{10}$

Neste sentido, este estudo que objetivou comparar o tratamento da terapia manual associada ao ultrassom, com o tratamento da cinesioterapia convencional associada ao laser de arseneto de galium (AsGa) na redução da dor e aumento da autonomia funcional, observou, em seus resultados, que ambas as terapias foram benéficas, significativamente $(p<0,05)$ na melhora da autonomia funcional e do quadro álgico de idosos com gonartrose.

$\mathrm{Na}$ presente pesquisa, foram utilizados dois recursos elétricos em cada um dos grupos: o ultrassom pulsátil e o laser de AsGa. Em uma revisão sistemática de 23 estudos randomizados controlados, o ultrassom foi encontrado para ter efeitos benéficos estatisticamente significativos $(\mathrm{p}<0,05)$ no tratamento da epicondilite lateral. ${ }^{24}$ Em 2003, a colaboração Cocbrane realizou uma revisão de ensaios utilizando técnicas de fisioterapia para distúrbios do ombro, que são comuns na população geriátrica, tanto quanto complicações no joelho. Em seus resultados, a terapia com laser de AsGa foi mais eficaz do que o placebo para a capsulite adesiva, e tanto o ultrassom pulsátil como o contínuo apresentaram melhora em comparação com o grupo placebo. ${ }^{25}$ Tais resultados possuem a mesma tendência daqueles expostos nos achados desta pesquisa.

O estudo de Perlman et al. ${ }^{26}$ mostrou que a técnica de manipulação foi significativamente melhor do que nenhuma intervenção para a redução da dor e melhora da funcionalidade em indivíduos com leve a moderada osteoartrite do joelho. A dor foi medida em cada $100 \mathrm{~mm}$ da escala visual analógica (EVA) e a diferença das médias entre os grupos, no intervalo de oito semanas, foi de 20,62 milímetros a favor da terapia manual, resultando num tamanho do efeito de 0,86 [95\% CI 0,37;1,36]. Achados semelhantes foram encontrados no estudo de Pollard et al. ${ }^{27}$ e Hoeksma et al. ${ }^{28}$ Estes dados corroboram os resultados encontrados pelo GE1, o qual revelou redução significativa $(\mathrm{p}<0,001)$ da dor após a aplicação da terapia de Mulligan associado ao ultrassom.

Grande parte dos estudos da mesma natureza do atual utilizou o mesmo período de tempo para a intervenção (oito semanas). ${ }^{29-31}$

A recomendação para encaminhar os pacientes com gonartrose sintomático para a fisioterapia é apoiada pelos resultados de três estudos clínicos randomizados, os quais revelaram, no pós-teste, melhora do quadro álgico, da função física, da qualidade de vida e do Western Ontario and McMaster Universities Osteoarthritis Index. Embora a eficácia dos achados, os resultados das investigações de Quilty et al..$^{32}$ e Bennell et al. ${ }^{33}$ contrapõem-se aos resultados em relação à melhora do quadro álgico.

A autonomia funcional em idosos, avaliada por meio do protocolo do GDLAM, tem sido reportada em diversos estudos com dança de salão, ${ }^{34}$ programa de recreação e caminhada,${ }^{35}$ treinamento funcional, ${ }^{36}$ treinamento muscular inspiratório, ${ }^{37,38}$ treinamento de força muscular, ${ }^{39}$ com programa de atividade física do programa de saúde da família, ${ }^{40}$ pilates ${ }^{41}$ e hidroginástica. ${ }^{42}$ Em todos estes, independentemente do tipo de treinamento ou tratamento realizado, observouse aumento no índice de GDLAM no pós-teste dos idosos pertencentes ao grupo experimental. 
Este estudo teve como limitações a ausência de um grupo controle e um "n amostral" pequeno no grupo experimental 2.

\section{CONCLUSÃO}

O presente estudo, com um poder do experimento de $98 \%$, inferiu que em idosos com gonatrose, a cinesioterapia associada à utilização do aparelho laser de arseneto de galium (AsGa) e a terapia manual (Mulligan) associada ao ultrassom

\section{REFERÊNCIAS}

1. Brandt KD, Dieppe P, Radin E. Etiopathogenesis of osteoarthritis. Med Clin North Am 2009;93(1):1-24.

2. Zhang W, Doherty M, Peat G, Bierma-Zeinstra MA, Arden NK, Bresnihan, et al. EULAR evidencebased recommendations for the diagnosis of knee osteoarthritis. Ann Rheum Dis 2010;69(3):483-9.

3. Zhang W, Moskowitz RW, Nuki G, Abramson $\mathrm{S}$, Altman RD, Arden N, et al. OARSI recommendations for the management of hip and knee osteoarthritis, Part II: OARSI evidence-based, expert consensus guidelines. Osteoarthr Cartil 2008;16(2):137-62

4. Zhang W, Nuki G, Moskowitz RW, Abramson S, Altman RD, Arden N, et al. OARSI recommendations for the management of hip and knee osteoarthritis: part III: Changes in evidence following systematic cumulative update of research published through January 2009. Osteoarthr Cartil 2010;18(4):476-99.

5. Jacox AK, Carr DB, Chapman CRea. Acute pain management: operative or medical procedures and trauma clinical practice guideline. Rockville (MD)7 US: Department of Health and Human Services. Agency for Health Care Policy and Research 1992:92-0032.

6. Recommendations for the medical management of osteoarthritis of the hip and knee: 2000 update. American College of Rheumatology Subcommittee on Osteoarthritis Guidelines. Arthritis Rheum 2000;43(9):1905-15. pulsátil beneficiam, de forma significativa, o aumento da autonomia funcional e a redução do quadro álgico.

Importante que novos estudos controlados sejam realizados, com uma diversificação de associação de técnicas, a fim de verificar a abrangência de recursos compatíveis com o tratamento da gonartrose, sendo adaptado em cada disponibilidade de ambiente e adaptação do paciente.
7. Pendleton A, Arden N, Dougados M, Doherty M, Bannwarth B, Bijlsma JWJ, et al. EULAR recommendations for the management of knee osteoarthritis: report of a task force of the Standing Committee for International Clinical Studies Including Therapeutic Trials (ESCISIT). Ann Rheum Dis 2000;59(12):936-44.

8. Lipsky PE. Algorithms for the diagnosis and management of musculoskeletal complaints. Introduction. Am J Med 1997;103(Suppl 6A):1S-2S.

9. Lee JA. Adult degenerative joint disease of the knee. Maximizing function and promoting joint health. Institute for Clinical Systems Integration. Postgrad Med 1999;105(7):183-6, 189-90, 94 passim.

10. Perret DM, Rim J, Cristian A. A geriatrician's guide to the use of the physical modalities in the treatment of pain and dysfunction. Clin Geriatr Med 2006;22(2):331-54.

11. Adie S, Naylor JM, Harris IA. Cryotherapy after total knee arthroplasty a systematic review and meta-analysis of randomized controlled trials. J Arthroplasty 2010;25(5):709-15.

12. French HP, Brennan A, White B, Cusack T. Manual therapy for osteoarthritis of the hip or knee: a systematic review. Man Ther 2011;16(2):109-17.

13. Brantingham JW, Globe G, Pollard H, Hicks M, Korporaal C, Hoskins W. Manipulative therapy for lower extremity conditions: expansion of literature review. J Manipulative Physiol Ther 2009;32(1):53-71. 
14. Bashaw R, Tingstad E. Rehabilitation of the Osteoarthritic Patient: Focus on the Knee. Clinics in Sports Medicine 2005;24:101-31.

15. Marfell-Jones M, Olds T, Stewart A, Carter L. ISAK: International standards for anthropometric assessment. South Africa: Potchefstroom; 2006.

16. Spilã S, Multanen J, Kallinen M, Era P, Suominen $\mathrm{H}$. Effects of strength and endurance training on isometric muscle strength and walking speed in elderly women. Acta Physiol Scand 1996;156(4):457-64.

17. Guralnik M, Simonsick EM, Ferrucci L, Glynn RJ, Berkman LF, Blazer DG, et al. A short physical performance battery assessing lower extremity function: association with self-reported disability and prediction of mortality and nursing home admission. J Gerontol 1994;49(2):M85-94.

18. Alexander NB, Ulbrich J, Raheja A, Channer D. Rising from the floor in older adults. Am Geriatr Soc J 1997;45(5):564-9.

19. Vale RGS, Pernambuco CS, Novaes JS, Dantas EHM. Teste de autonomia funcional: vestir e tirar uma camiseta (VTC). Rev Bras Cienc Mov 2006;14(3):71-8.

20. Andreotti R, Okuma SS. Validação de uma bateria de testes de atividades da vida diária para idosos fisicamente independentes. Rev Paul Educ Fís 1999;13(1):46-66.

21. Vale RGS. Avaliação da autonomia funcional do idoso. Fit Perf J 2005;4:4.

22. Borg G, editor. Borg's Perceived Exertion and Pain Scales. Champaign, IL: Human Kinetics; 1998.

23. Schneider H, Cristian A. Role of rehabilitation medicine in the management of pain in older adults. Clin Geriatr Med 2008;24(2):313-34, vii.

24. Smidt N, Assendelft WJ, Arola H, Malmivaara A, Greens S, Buchbinder R, et al. Effectiveness of physiotherapy for lateral epicondylitis: a systematic review. Ann med 2003;35(1):51-62.

25. Green S, Buchbinder R, Hetrick SE. Physiotherapy interventions for shoulder pain. In: Cochrane Database Syst Rev[Internet]. Hoboken, NJ: John Wiley \& Sons, Ltd. c2009 - . 2 p. Disponível em: http://cochrane.bvsalud.org/doc.php?db=reviews\&id $=\mathrm{CD} 004258 \& \mathrm{lib}=\mathrm{COC}$

26. Perlman AI, Sabina A, Williams AL, Njike VY, Katz DL. Massage therapy for osteoarthritis of the knee: a randomized controlled trial. Arch intern med 2006;166(22):2533-8.
27. Pollard H, Ward G, Hoskins W, Hardy K. The effect of a manual therapy knee protocol on osteoarthritic knee pain: a randomised controlled trial. J Can Chiropr Assoc 2008;52(4):229-42.

28. 28. Hoeksma HL, Dekker J, Ronday HK, Heering A, Van der Lubbe N, Vel C, et al. Comparison of manual therapy and exercise therapy in osteoarthritis of the hip: a randomized clinical trial. Arthritis Rheum 2004;51(5):722-9.

29. Hughes SL, Seymour RB, Campbell R, Pollak N, Huber $G$, Sharma L. Impact of the fit and strong intervention on older adults with osteoarthritis. Gerontologist 2004;44(2):217-28.

30. Hughes SL, Seymour RB, Campbell RT, Huber G, Pollak N, Sharma L, et al. Long-term impact of Fit and Strong! on older adults with osteoarthritis. Gerontologist 2006;46(6):801-14.

31. Callahan LF, Mielenz T, Freburger J, Shreffler J, Hootman J, Brady T, et al. A randomized controlled trial of the people with arthritis can exercise program: symptoms, function, physical activity, and psychosocial outcomes. Arthritis Rheum 2008;59(1):92-101.

32. Quilty B, Tucker M, Campbell R, Dieppe P. Physiotherapy, including quadriceps exercises and patellar taping, for knee osteoarthritis with predominant patello-femoral joint involvement: randomized controlled trial. J Rheumatol 2003;30(6):1311-7.

33. Bennell KL, Hinman RS, Metcalf BR, Buchbinder R, McConnell J, McColl G, et al. Efficacy of physiotherapy management of knee joint osteoarthritis: a randomised, double blind, placebo controlled trial. Ann Rheum Dis 2005;64(6):906-12.

34. Borges EG, Cader SA, Vale RG, Cruz TH, Carvalho $\mathrm{MC}$, Pinto FM, et al. The effect of ballroom dance on balance and functional autonomy among the isolated elderly. Arch Gerontol Geriatr 2012;55(2):492-6.

35. Fraga MJ, Cader SA, Ferreira MA, Giani TS, Dantas EHM. Aerobic resistance, functional autonomy and quality of life (QoL) of elderly women impacted by a recreation and walking program. Arch Gerontol Geriatr 2011;52(1):e40-3.

36. Leal SMO, Borges EGS, Fonseca MA, Alves Junior ED, Cader S, Dantas EHM. Efeitos do treinamento funcional na autonomia funcional, equilíbrio e qualidade de vida de idosas. Rev Bras Ciênc Mov 2010;17(3):61-9. 
37. Fonseca MD, Cader SA, Dantas EHM, Bacelar SC, da Silva EB, Leal SMD. Respiratory Muscle Training Programs: Impact on the Functional Autonomy of the Elderly. Ver Assoc Med Bras 2010;56(6):642-8.

38. Cader SA, Vale RG, Castro JC, Bacelar SC, Biehl C, Gomes MC, et al. Inspiratory muscle training improves maximal inspiratory pressure and may assist weaning in older intubated patients: a randomised trial. J Physiother 2010;56(3):171-7.

39. da Silva JG, Cader SA, Dopico X, Iglesias Soler E, Martin Dantas EH. [Strength training, level of muscular strength and functional autonomy in a population of elderly women]. Rev Esp Geriatr Gerontol 2009;44(5):256-61. Espanhol.
40. Guimarães AC, Rocha CAQC, Gomes ALM, Cader SA, Dantas EHM. Efeitos de um programa de atividade física sobre o nível de autonomia de idosos participantes do programa de saúde da família. Fitness \& Perform J 2008;7(1):5-9.

41. Rodrigues BGS, Cader SA, Torres NVOB, Oliveira EM, Dantas EHM. Autonomia funcional de idosas praticantes de Pilates. Fisioter Pesq 2010;17(4):300-5.

42. Belloni D, Albuquerque A, Rodrigues T, Mazini Filho M, Silva V. Estudo comparativo entre a autonomia funcional de mulheres idosas praticantes e não praticantes de hidroginástica. Rev Educ Fís 2008;140:20-6. 